\title{
Ensemble coding remains accurate under object and spatial visual working memory load
}

\author{
Michael L. Epstein ${ }^{1}$ • Tatiana A. Emmanouil ${ }^{1,2}$
}

Published online: 9 June 2017

(C) The Psychonomic Society, Inc. 2017

\begin{abstract}
A number of studies have provided evidence that the visual system statistically summarizes large amounts of information that would exceed the limitations of attention and working memory (ensemble coding). However the necessity of working memory resources for ensemble coding has not yet been tested directly. In the current study, we used a dual task design to test the effect of object and spatial visual working memory load on size averaging accuracy. In Experiment 1, we tested participants' accuracy in comparing the mean size of two sets under various levels of object visual working memory load. Although the accuracy of average size judgments depended on the difference in mean size between the two sets, we found no effect of working memory load. In Experiment 2, we tested the same average size judgment while participants were under spatial visual working memory load, again finding no effect of load on averaging accuracy. Overall our results reveal that ensemble coding can proceed unimpeded and highly accurately under both object and spatial visual working memory load, providing further evidence that ensemble coding reflects a basic perceptual process distinct from that of individual object processing.
\end{abstract}

Keywords Ensemble coding · Working memory

A fundamental question in the study of vision is how we are able to effectively process the overwhelming amount of detail

Michael L. Epstein

mepstein@gradcenter.cuny.edu

1 Program in Psychology, The Graduate Center of the City University of New York, 365 5th Avenue, New York, NY 10016, USA

2 Department of Psychology, Baruch College of the City University of New York, 55 Lexington Avenue, New York, NY 10010, USA that surrounds us, in light of the many well-studied limitations of attention and working memory (Cowan, 2001; Luck \& Vogel, 1997; Posner, 1980; Pylyshyn \& Storm, 1988; Rensink, O'Regan, \& Clark, 1996). A number of studies have proposed that the discrepancy may be explained by the ability of the human visual system to rapidly summarize properties of scenes without encoding individual objects (Alvarez, 2011; Ariely, 2001; Chong \& Treisman, 2003). For example, the visual system may encode groups of similar objects, such as leaves on a tree or pebbles on the beach, in terms of their average as opposed to their individual properties (ensemble coding). In fact, several studies suggest that the visual system can encode averages in several dimensions such as size (Ariely, 2001; Chong \& Treisman, 2003), speed and direction of motion (Watamaniuk \& Duchon, 1992; Williams \& Sekuler, 1984), orientation (Parkes, Lund, Angelucci, Solomon, \& Morgan, 2001), color (Maule, Witzel, \& Franklin, 2014) and even facial expression and emotion (Haberman \& Whitney, 2007, 2009).

An interesting aspect of ensemble coding is that it occurs rapidly and efficiently for groups of objects whose number exceed the capacity limits of focused attention and working memory (Alvarez \& Oliva, 2008; Chong \& Treisman, 2003). Judgments of the mean remain accurate despite rapid presentation of stimuli (Chong \& Treisman, 2003; Whiting \& Oriet, 2011), time delays during presentation (Chong \& Treisman, 2003), varied spatial distributions of objects (Chong \& Treisman, 2005b), and varied heterogeneity of items within a set (Chong \& Treisman, 2003; Utochkin \& Tiurina, 2014). While there remains debate as to possible limitations in dividing attention between sets or dimensions in statistical processing (Attarha \& Moore, 2015a, 2015b; Attarha, Moore, \& Vecera, 2014; Chong \& Treisman, 2005b; Emmanouil \& Treisman, 2008), the high accuracy of statistical processing within sets suggests that ensemble coding operates in a manner distinct from that of the perception of individual objects. 
Furthermore, attentional demands of ensemble coding are very different from those of individual object processing. The evidence so far suggests that although statistical perception requires attentional resources, it relies on a broad distribution of attention rather than focused attention to each item. A recent study showed that some amount of attention is necessary for ensemble coding by demonstrating that if attention is engaged on a demanding task away from the set, ensemble properties can go entirely unnoticed (Jackson-Nielsen, Cohen, \& Pitts, 2017). Also, Huang (2015) observed a similar cost of dividing attention between individual objects and perceptual groups, suggesting that statistical properties require as much attention as individual features. At the same time, several studies suggest that a minimal amount of attention spread over the scene is sufficient to process statistical information (Alvarez \& Oliva, 2008; Chong \& Treisman, 2005a; Joo, Shin, Chong, \& Blake, 2009), even when it is not sufficient for conscious awareness of individual features within the set (Ward, Bear, \& Scholl, 2016; but see Bronfman, Brezis, Jacobson, \& Usher, 2014). This is further supported by clinical studies showing that even patients with unilateral spatial neglect can use information from their neglected visual field when computing statistical information (Pavlovskaya, Soroker, Bonneh, \& Hochstein, 2015; Yamanashi Leib, Landau, Baek, Chong, \& Robertson, 2012). Taken together, these results suggest that ensemble coding operates independently of focused attention and working memory limitations, instead using a distinct distributed attention mechanism, perhaps as part of a rapid feedforward pass of visual information which precedes detailed object processing through feedback connections (Hochstein \& Ahissar, 2002; Lamme \& Roelfsema, 2000; Treisman, 2006).

However, some studies question the claim that ensemble coding bypasses limitations of focused attention and working memory. A number of studies have suggested that observed accuracy in ensemble coding tasks could be achieved using various strategies involving individual object processing (Allik, Toom, Raidvee, Averin, \& Kreegipuu, 2013; Maule \& Franklin, 2016; Myczek \& Simons, 2008), although the degree to which these strategies are actually used by participants remains unclear (Ariely, 2008; Chong, Joo, Emmanouil, \& Treisman, 2008). It has also been argued that average estimates can be skewed by salient individual objects (De Fockert \& Marchant, 2008) or unusual distributions (Marchant, Simons, \& De Fockert, 2013; but see Utochkin \& Tiurina, 2014), which would suggest that individual object processing precedes and biases perception of the set as a whole. Therefore, while it is generally agreed upon that statistical summaries can be calculated quickly and efficiently, the extent to which ensemble coding relies on focused attention and working memory remains debated.

In the present study, we tested the role of working memory in ensemble coding using a dual task paradigm. Specifically we asked participants to estimate mean sizes while maintaining items in working memory. Similar paradigms have been previously used to examine the role of working memory in visual search (Woodman \& Luck, 2004; Woodman, Vogel, \& Luck, 2001). If ensemble coding relies on the encoding of individual items, it would require working memory resources for the storage and manipulation of these items and would become less efficient under working memory load. We tested this prediction in two experiments, investigating the effects of object (Experiment 1) and spatial (Experiment 2) working memory load on the accuracy of ensemble coding.

\section{Experiment 1}

In Experiment 1 we tested the effect of object working memory load on the accuracy of ensemble coding using a dual task where participants were required to perform an average size comparison between two groups of white circles while maintaining an array of colored squares in working memory. By comparing accuracy in the size averaging task across low, high, and no working memory load, we tested if working memory resources are required for accurate ensemble coding.

\section{Method}

Participants Twelve participants (eight female, 10 righthanded, average age 22 years) participated in this experiment for course credit. All participants had normal or corrected-tonormal vision and gave written informed consent.

Stimuli Stimuli were generated using the PsychoPy Python toolbox (Peirce, 2007). Stimuli were presented on a Dell CRT monitor set with a 75-Hz refresh rate and a screen resolution of $1280 \times 1084$. All stimuli were displayed on a gray background with a centered fixation cross $0.75^{\circ}$ across in size.

The working memory task was largely adapted from Woodman et al. (2001). Their experiment used a simple dual task design in which participants performed a visual search task while maintaining two or four items in working memory. In our design, we substituted a size averaging task for the visual search task used by Woodman et al. (2001) to test for the effects of working memory load on ensemble coding accuracy. A key difference in our design was that memory object locations in this task were distributed widely across the screen to discourage participants from focusing attention on small regions of the display. Squares were $0.65^{\circ}$ across in size and were randomly assigned a color (red, blue, violet, green, yellow, black, white, or orange). Each individual object within a memory load set was assigned a unique color. Squares were randomly assigned a location on an invisible 2 $\times 4$ grid on the left and right side of fixation, with each 
possible location separated by $4^{\circ}$. Grids were each placed $2^{\circ}$ away from fixation. Square locations were also controlled so that in each condition an even number of squares appeared on each side of the screen (one on each side for low load, and two on each side for high load). Once squares were assigned a grid position, location was further jittered up to $1^{\circ}$ in any direction. For the visual memory test squares were placed in identical positions as the original display, with a $50 \%$ chance that one changed to a new color. Colors were never repeated in a display.

For the size averaging task, two sets of 12 objects each were displayed on the left and right side of fixation. Objects within these sets were displayed in random locations on invisible $3 \times 4$ grids with a cell size of $3^{\circ}$, and were jittered in location a further $1^{\circ}$ in any direction to add spatial randomness and encourage perception of the objects as a natural set as opposed to an artificial arrangement. Object grids on each side were $4^{\circ}$ away from fixation. Sets consisted of four groups of three identical objects each, with each group $25 \%$ apart in size, measured in diameter (i.e., groups with diameters $1.0^{\circ}, 1.25^{\circ}$, $1.56^{\circ}$, and $1.95^{\circ}$ ). A second set was created to be $5 \%, 10 \%$, $15 \%$, or $20 \%$ larger in mean using the following method: First, a set was created in which items were $5 \%, 10 \%, 15 \%$, or $20 \%$ larger than in the first set. Then, to discourage participants from simply comparing the extreme (largest or smallest) individual items across sets, we equated the largest items in the two sets on half the trials and the smallest items in the other half. Finally, the remaining items in the set were adjusted equally to maintain the original average difference between sets. Additionally, to increase the diversity of stimuli being presented while maintaining the same average size differences, on each trial the size of items in both sets were equally increased by a random factor $(0 \%, 5 \%, 10 \%, 15 \%$, or $20 \%)$. The display side for the larger set was randomized.

Task Participants were briefly presented with two (low working memory load) or four (high load) colored squares. While retaining these objects in working memory, two sets of white circles of varied sizes were flashed on the left and right side of fixation, and participants indicated which set appeared to contain objects that were on average larger. After a brief delay, the original colored squares reappeared with a $50 \%$ chance that one had changed color, and participants indicated if they noticed a change or not compared to the original presentation. In one third of the trials, there was no memory array, and participants only performed the size averaging task (no load condition). Note that different features (size and color) were used in the memory and ensemble coding tasks to ensure any interference would be due solely to the inability to perform the two tasks together rather than a bias induced by the contents of working memory on perception of the set arrays (see Soto, Hodsoll, Rotshtein, \& Humphreys, 2008, for an example of this possible confound).
To control for use of verbal working memory, during all conditions, participants were required to perform an articulatory suppression task where they repeated the letters $A B C D$ continuously throughout trials (Besner, Davies, \& Daniels, 1981). An experimenter sat within the testing booth to monitor that participants continually performed articulatory suppression. No participant was removed from the study for inadequate articulatory suppression.

Procedure Figure 1 shows an example trial in this experiment. Trials began with the visual memory array presented for 1,000 $\mathrm{ms}$, followed by an interstimulus interval (ISI) of $1,000 \mathrm{~ms}$, then the presentation of the circle arrays for $200 \mathrm{~ms}$. Following a second 2,000 ms ISI, the test array was displayed for 2,000 ms. The intertrial interval (ITI) was $4,000 \mathrm{~ms}$. For the first $3,000 \mathrm{~ms}$ of the ITI, the fixation cross was removed, leaving the screen blank, and during the final $1,000 \mathrm{~ms}$, the fixation cross reappeared to alert the participant that the next trial was about to begin. For trials in the no load condition, memory items were simply not displayed, resulting in a lengthened ISI but maintaining identical trial lengths to the low and high load conditions.

Participants responded using the left and right arrow keys for both tasks. For the size averaging task, participants indicated which side of the screen (left or right) contained the larger items on average by pressing the left and right arrow keys, respectively. Participants' responses to the averaging task were recorded during presentation and during the 2,000 ms ISI to the test working memory array. For the working memory task, half of the participants pressed the left arrow key to signify identical and the right arrow to signify change. This was switched for the remaining participants. Responses for this task were recorded for the $2,000 \mathrm{~ms}$ that the test array was displayed.

Working memory load varied between low, high, and no working memory load, while the possible difference in mean size for the averaging task was $5 \%, 10 \%, 15 \%$, or $20 \%$. The full experiment consisted of 240 total trials: 20 trials for each combination of load and difference in means. Trials were presented in random order, to discourage the use of different strategies between conditions. Participants were allowed to take a brief break every 40 trials to prevent fatigue, resulting in six separate blocks. Before the task, participants engaged in practice sessions consisting of one trial for each condition (12 trial blocks). During practice, participants received feedback on each trial indicating if their responses were correct or incorrect. Feedback was provided only in practice trials to ensure task competence and confidence; it was not used during the experimental trials. Participants performed, at most, four practice blocks.

Analysis Descriptive statistics were calculated using MATLAB, and ANOVAs were run with SPSS. Accuracy in the two tasks (working memory, size averaging) was calculated for each memory load and average difference between sets. Ensemble coding 


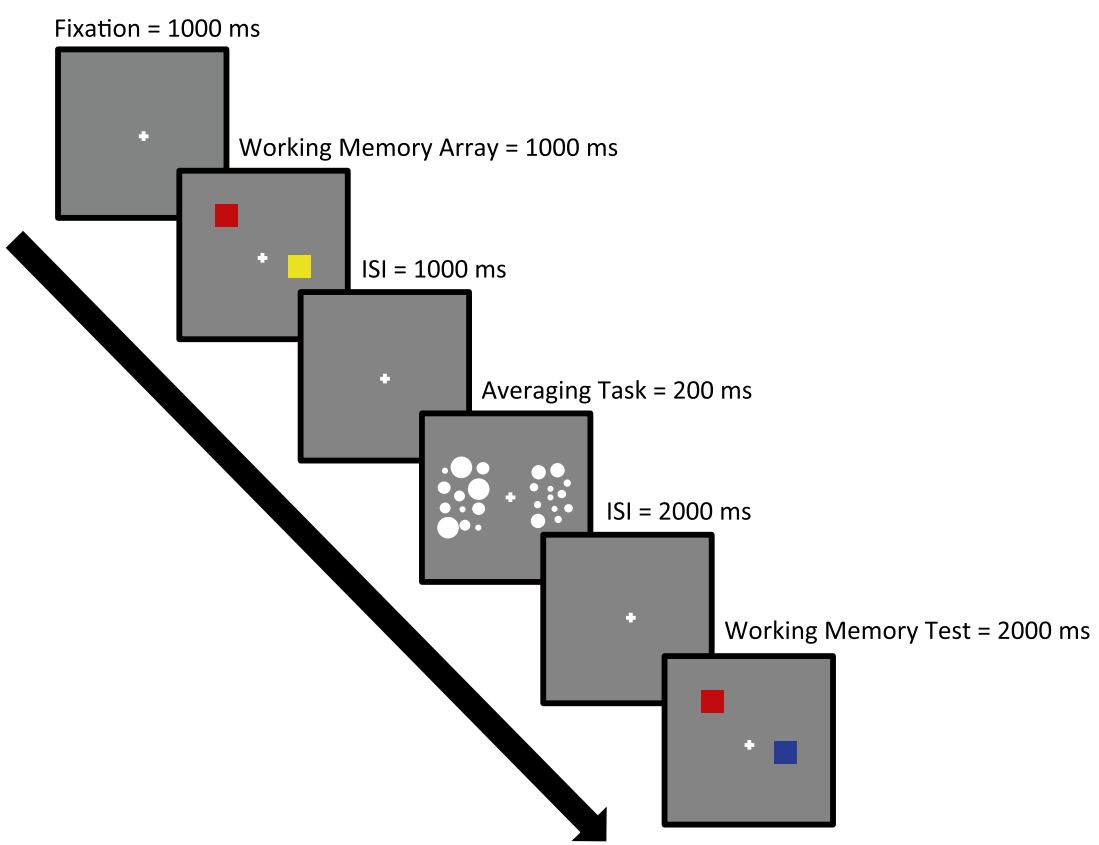

Fig. 1 Example of task with low working memory load of two items. Sizes adjusted for clarity. (Color figure online)

accuracy was submitted to a $3 \times 4$ ANOVA with working memory load (high, low, none) and average difference between sets $(5 \%, 10 \%, 15 \%$, and $20 \%)$ as within-subject factors. Working memory accuracy was submitted to a $2 \times 4$ ANOVA with working memory accuracy (high, low) and average difference between sets $(5 \%, 10 \%, 15 \%$, and $20 \%)$ as within subject factors. Greenhouse-Geisser corrections were applied in conditions where violations of sphericity were detected. Ensemble coding accuracy was further subjected to a Bayes factor analysis to directly compare models of the null and alternative hypotheses, using the BayesFactor package in R (Rouder, Morey, Speckman, \& Province, 2012).

\section{Results}

Figures 2 and 3 show accuracy results for ensemble coding and object visual working memory. While accuracy in the averaging task increased as the average difference between sets increased, $F(1.682,18.506)=16.816, p<.001, \eta_{p}^{2}=$ 0.60 , there was no effect of memory load on accuracy, $F(2$, $22)=.417, p=.664, \eta_{p}^{2}=0.01$, and no interaction, $F(6,66)=$ $.603, p=.727, \eta_{p}^{2}=0.05$. Accuracy in the visual memory task was affected by memory load, $F(1,11)=21.949, p=.001, \eta_{p}^{2}$ $=0.67$, but showed no difference based on average difference between sets, $F(3,33)=1.486, p=.236, \eta_{p}^{2}=0.12$, and no interaction, $F(3,33)=.454, p=.716, \eta_{p}^{2}=0.04$.

It should be acknowledged that while these results support our hypothesis, null findings in standard null hypothesis significance testing (NHST) solely report a failure to reject the null hypothesis, rather than giving an indication of how strongly the null hypothesis can be supported (Wilkinson, 1999; also see J. Cohen, 1994, for a broader discussion of issues with NHST). Bayes factor analyses, however, allow for a direct comparison of the likelihood of models of the null and the alternative hypothesis, allowing for more substantial support of the null hypothesis (Masson, 2011). A Bayes factor analysis of the effect of working memory load on averaging accuracy resulted in $K=.0782 \pm 0.84 \%$, providing stronger support for the null compared to the alternative hypothesis (Jeffreys, 1961; Kass \& Raftery, 1995).

\section{Discussion}

Supporting the hypothesis that ensemble coding operates outside of limitations of working memory, our results show no effect of working memory load on averaging accuracy. Averaging accuracy significantly increased as mean difference between sets increased, indicating that the task was appropriately difficult to capture varying levels of ensemble coding performance. Similarly, as expected, working memory accuracy significantly decreased as load increased. However, memory load, even with four items thought to exhaust working memory capacity (Alvarez \& Cavanagh, 2004; Luck \& Vogel, 1997), had no effect on averaging accuracy. Similarly, accuracy in the memory task was not impacted by the difficulty of the size averaging task. Despite high difficulty levels within tasks, participants were still able to perform both tasks simultaneously.

These findings support a separation between working memory and ensemble coding. If estimation of set properties 


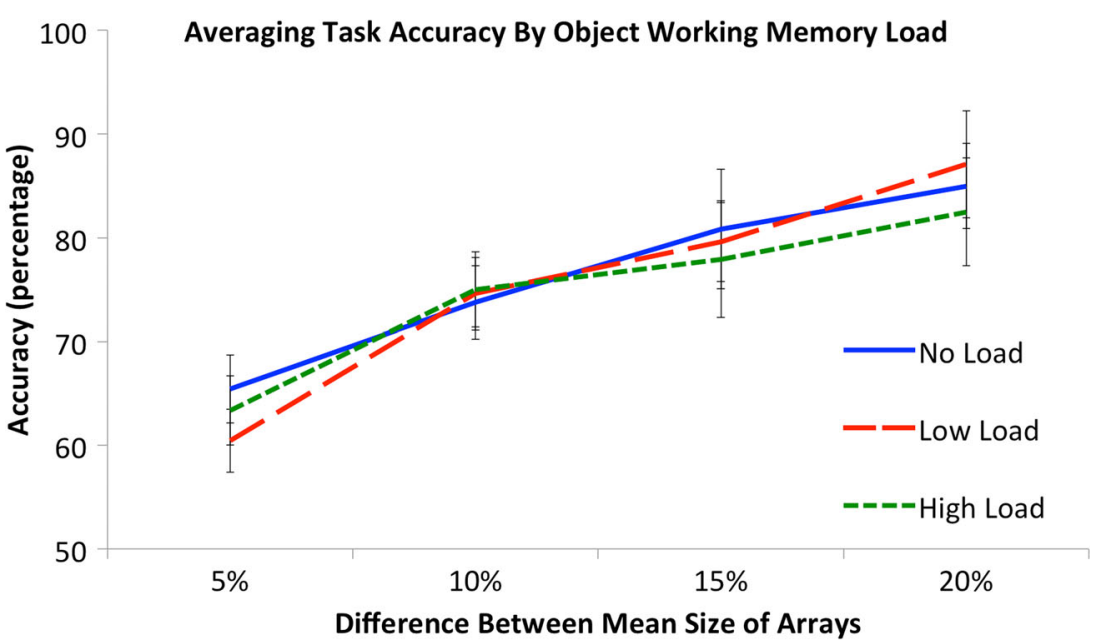

Fig. 2 Accuracy in averaging task for all averaging difficulty and object working memory load conditions. Error bars represent the standard error of the mean. (Color figure online)

involved the sampling of individual objects, these items would require storage in working memory as they were sequentially processed and compared. Rather, even in conditions where working memory is full, comparisons of the average size of sets of objects can be made with high accuracy, even when their number far exceeds the limitations of individual object processing. These results support the hypothesis that ensemble coding involves a rapid, parallel processing of whole groups, resulting in a perception of solely the average, as opposed to some number of individual objects.

\section{Experiment 2}

While the results of Experiment 1 provide evidence that ensemble coding can proceed despite object working memory load, it is unclear if this result would generalize to spatial working memory load. Spatial working memory resources are thought to be distinct from those of object working memory, in that storage of locations involves attentional rehearsal of target locations (Awh \& Jonides, 2001; Awh, Jonides, \& Reuter-Lorenz, 1998). Here, a similar dual task to Experiment 1, using instead spatial working memory load, is employed to test if attentional rehearsal of spatial locations causes a reduction in accuracy in ensemble coding.

\section{Method}

Participants Twelve participants (seven female, 10 righthanded, one left-handed, one ambidextrous, average age 21.75 years) participated in this experiment for course credit. All participants had normal or corrected-to-normal vision. No participant in Experiment 2 had participated in Experiment 1. All participants gave written informed consent.

Stimuli Ensemble coding stimuli were identical to that in Experiment 1 . Working memory arrays were the same as in Experiment 1, except the squares appeared in white instead of

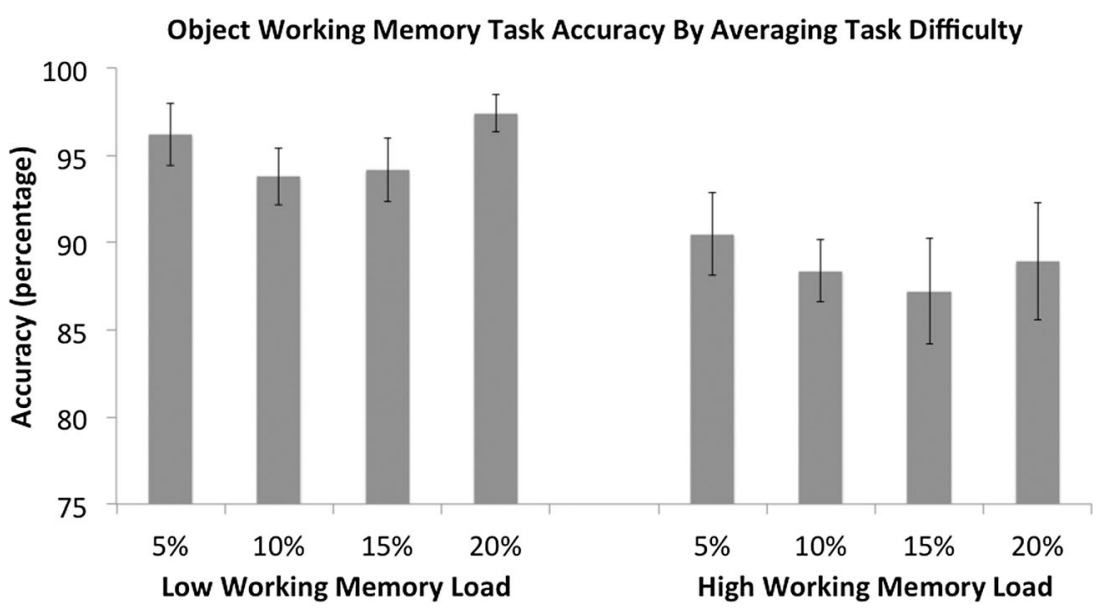

Fig. 3 Accuracy in the low and high load object working memory conditions for each averaging task difficulty condition. Error bars represent the standard error of the mean 
in color. Additionally, during the memory test display, in 50\% of trials, one square was moved to a new random location on the grid, with the restriction that it remained on the same side of the screen it was originally displayed on. In the remaining trials the squares appeared in the same location as in the original presentation.

Task This task was designed to be similar to that of Experiment 1, but with spatial working memory load replacing object working memory load. Participants were instructed to remember the locations of two (low load) or four (high load) white squares on the screen. The size averaging task was identical to that of Experiment 1. After the averaging task, participants viewed the test array, in which the squares reappeared with a $50 \%$ chance that one object was moved to a randomly selected and unused grid position on the same side of the screen. To maintain consistency, participants were again required to perform the same articulatory suppression task used in Experiment 1.

Procedure Procedure was identical to that of Experiment 1, with spatial working memory load replacing object-based working memory load.

Analysis Behavioral analysis was again performed using MATLAB and SPSS. Ensemble coding accuracy was submitted to a $3 \times 4$ ANOVA, with spatial working memory load (high, low, none) and average difference between sets (5\%, $10 \%, 15 \%$, and $20 \%$ ) as within-subject factors. Spatial working memory accuracy was tested in a $2 \times 4$ ANOVA, with working memory accuracy (high, low) and average difference between sets $(5 \%, 10 \%, 15 \%$, and $20 \%)$ as within-subject factors. The effect of spatial memory load on averaging accuracy was again subjected to a Bayes factor analysis.

\section{Results}

Figures 4 and 5 show accuracy results in the size averaging and memory tasks. Accuracy in the size averaging task was affected by difficulty, $F(3,33)=41.767, p<.001, \eta_{p}^{2}=0.79$, but there was no effect of spatial memory load, $F(6,66)=$ $.813, p=.564, \eta_{p}^{2}=0.04$, and no interaction, $F(6,66)=$ $.813, p=.564, \eta_{p}^{2}=0.07$. Again, to provide support for the null hypothesis, a Bayes factor analysis was performed resulting in $K=0.084 \pm 1.09 \%$, indicating support for the null hypothesis compared to the alternative hypothesis (Jeffreys, 1961; Kass \& Raftery, 1995). Accuracy in the spatial memory task was affected by memory load, $F(1,11)=5.619, p=.037$, $\eta_{p}^{2}=0.34$, but there was again no effect of average difference between sets, $F(3,33)=.136, p=.938, \eta_{p}^{2}=0.01$, and no interaction, $F(3,33)=.850, p=.477, \eta_{p}^{2}=0.07$.

\section{Discussion}

Our results show no effect of spatial working memory load on ensemble coding accuracy. Similar to the findings from Experiment 1, the two tasks were individually challenging, as shown by a significant effect of difficulty on accuracy within tasks. Nevertheless, both tasks could still be performed simultaneously.

These results further support that it is possible for ensemble coding to proceed without working memory resources and with only a minimal amount of distributed attention. While Chong and Treisman (2005a) reported that focused attention can cause a significant reduction in averaging accuracy, it is important to note that accuracy in their task was simply diminished but was not at chance. As the object locations in our task overlapped with the sets of objects to be averaged, it is possible that attention was still distributed efficiently enough simply attending to these locations. Furthermore, as the spatial task moved objects a great distance, participants may have adopted a strategy of using wider distributions of attention (see Alvarez \& Franconeri, 2007, for data supporting varying spatial resolution of attention in object tracking tasks). Another potential strategy for the memory task could involve spreading one's attention across the set of locations to be remembered, by attending to a line (for two objects) or rectangle (for four objects), which may ease the distribution of attention over the set of objects to be averaged. Though it is not possible to distinguish between these strategies within this paradigm, the finding remains that despite spatial memory load, attention can be distributed across the objects to be processed, allowing ensemble coding to proceed efficiently and accurately.

\section{General discussion}

Ensemble coding is generally understood to be rapid, accurate, and resistant to interference (Alvarez \& Oliva, 2008; Chong \& Treisman, 2003), however an important question is the extent to which attention and working memory systems are required for the accurate calculation of set properties. Experiment 1 tested the necessity of object working memory, while Experiment 2 investigated the role of spatial working memory resources in ensemble coding. The results of Experiment 1 revealed no effect of object working memory load on averaging accuracy, even when working memory was loaded to capacity. Similarly, accuracy on the working memory task was unaffected by averaging task difficulty. This provides evidence that both tasks could be performed simultaneously, despite clear evidence of within-task difficulty. The results of Experiment 2 were consistent with those of Experiment 1, showing no effect of spatial working memory load on averaging accuracy or of averaging difficulty on 


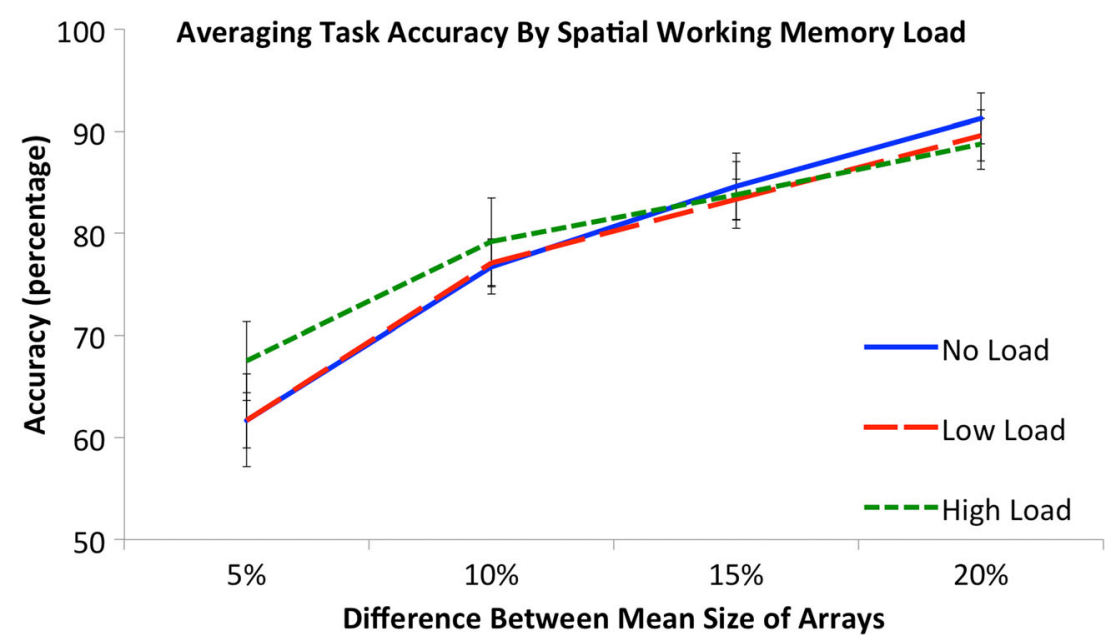

Fig. 4 Accuracy in averaging task for all averaging difficulty and spatial working memory load conditions. Error bars represent the standard error of the mean

spatial working memory performance. Altogether, these results signify that statistical properties can be perceived with limited working memory resources.

The current results corroborate the view that ensemble coding reflects a basic perceptual process wherein sets are processed as a whole (Treisman, 2006). Although accurate approximations of set properties could be achieved by randomly sampling just two or three representative items (Marchant et al., 2013; Myczek \& Simons, 2008), the evidence that set statistical properties can be perceived without the use of working memory suggests that this strategy is unlikely. It is more reasonable to hypothesize that ensemble coding and individual object processing can proceed separately and simultaneously, with each having the capability to inform the other. Some evidence of this can be seen in how items encoded into working memory can be biased by statistics automatically derived from irrelevant items within presented sets (Brady \& Alvarez, 2011) and in how salient items within a set can weigh perception of the overall average (De Fockert \& Marchant, 2008).
Although we provide a clear demonstration of ensemble coding under working memory load, we cannot rule out that working memory becomes necessary for statistical processing under different conditions. For example, one may hypothesize a greater need for storage of information in working memory if ensemble coding is performed over sequentially presented objects (Albrecht \& Scholl, 2010; Haberman, Harp, \& Whitney, 2009; Hubert-Wallander \& Boynton, 2015) or when the mean needs to be stored in working memory to be later compared with a probe (Ariely, 2001; Chong \& Treisman, 2005a). Similarly, future studies will need to test how ensemble coding and working memory tasks may interact, for example, whether items in working memory may influence the processing of ensembles similarly to how they can bias the processing of individual items in the visual field (Soto et al., 2008). Therefore, further studies will need to clarify the exact conditions under which summary statistics and working memory tasks may share resources or information. In any case, the current results provide evidence that ensemble coding can proceed independently of working memory demands, a result

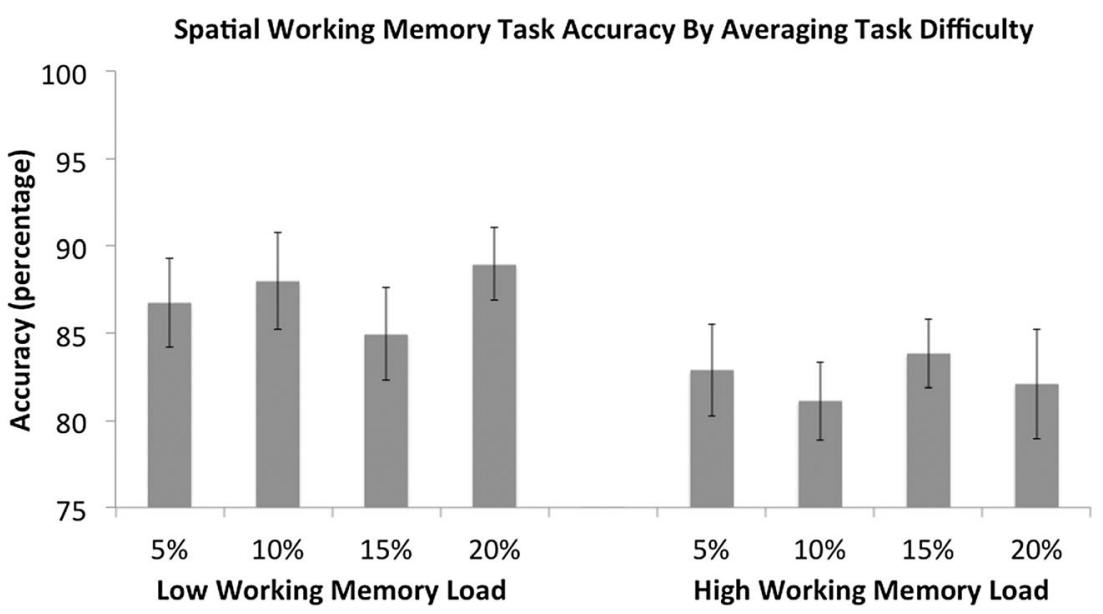

Fig. 5 Accuracy in the low and high spatial working memory load conditions for each averaging task difficulty condition. Error bars represent the standard error of the mean 
that informs our understanding of the basic mechanism and contributes to the debate over whether it involves individual object selection (Ariely, 2008; Chong et al., 2008; Myczek \& Simons, 2008).

While we instructed our participants to treat the two tasks as equally important, it is possible that they prioritized the working memory task over the size averaging task since the encoding phase of the working memory task preceded the presentation of the sets. If this were the case, it could be argued that the averaging task was performed with resources that were left unused by the working memory task (Huang, 2015). Although this possibility cannot be ruled out, it becomes less likely given the fact that ensemble coding was unaffected across different levels of load. If unused working memory resources were instrumental for the task, one would expect that the difference in available resources between high and low working memory load would be reflected in averaging task performance. Therefore, we would argue that if participants were prioritizing the working memory task, this would not necessarily explain the lack of effect of load on ensemble coding.

Our results are consistent with previous studies showing that sets of objects require similar working memory resources as individual objects (Im \& Chong, 2014; McNair, Goodbourn, Shone, \& Harris, 2016). Im and Chong (2014) found that the capacity for encoding the average sizes of multiple sets was close to the known capacity for memorizing individual objects, suggesting that statistical summaries are processed and condensed prior to storage in working memory. Additionally, a recent study using attentional blink found a similar decrement in $\mathrm{T} 2$ detection whether $\mathrm{T} 1$ consisted of an individual item or an ensemble (McNair et al., 2016). Therefore, our results corroborate and extend the abovementioned studies in suggesting that ensemble coding need not pose working memory demands prior to the storage of the mean.

Although we claim that ensemble coding does not rely on focused attention and working memory, our results are still consistent with the idea that ensemble coding requires a broad distribution of attention (Chong \& Treisman, 2005a). The lack of an effect in Experiment 2 may appear to stand in contradiction to Jackson-Nielsen et al.'s (2017) finding that when attention is solely engaged on a demanding task, participants become blind to changes in statistical properties outside of the focus of attention. It is critical to note, however, that while their study provided evidence that attention is required for ensemble coding, it also replicated prior results that very little residual attention is necessary to restore perception of ensemble properties (Bronfman et al., 2014; Ward et al., 2016). In the present experiments, the object and spatial working memory tasks were designed to avoid a narrow focus of attention that could incur a decrement to ensemble coding performance above and beyond working memory load. More specifically, the locations of objects in both the object and the spatial working memory tasks spanned a large window, encouraging a broad compared to a narrow focus of attention. Therefore, our results do not dispute the results of Jackson-Nielsen et al. (2017), which suggest that attention is necessary for ensemble coding.

Overall, our results support ensemble coding as a unique kind of processing, involving the spread of attention across a group of objects and the rapid acquisition of set properties. The visual world would be an amazingly stark place if we were restricted solely by the limitations of focused attention and individual object processing. The ability to use different ranges of attention as needed - distributed attention for calculating general statistical information and focused attention when detailed individual information is needed - can explain a great deal about how it is that the complex visual world can be summarized and then selectively processed in greater detail. Furthermore, the current study supports the view that the perception of set properties need not be disrupted when working memory is engaged. The results are consistent with previous evidence that, even as the spotlight of attention is sharply focused, stimuli in the periphery can still be processed to an extent that provides useful information (Alvarez \& Oliva, 2008). Altogether, these results cast new light on the sources of information that inform our rich experience of the visual environment (Cohen, Dennett, \& Kanwisher, 2016).

In conclusion, the present findings indicate that ensemble coding can occur with limited working memory resources. The potential interactions between ensemble coding and working memory - for example, how the contents of working memory may influence ensemble coding - require further study. Nevertheless, the current results support that idea that ensemble coding is a distinct perceptual mechanism, separable from that underlying individual object processing.

Author note This work was supported by PSC-CUNY Research Award TRADA 46-769.

Many thanks to Jennifer Dana, Philip Kang, and Keunwoo Park for their help in collecting data for this project.

\section{References}

Albrecht, A. R., \& Scholl, B. J. (2010). Perceptually averaging in a continuous visual world: Extracting statistical summary representations over time. Psychological Science: A Journal of the American Psychological Society, 21(4), 560-567. doi:10.1177/ 0956797610363543

Allik, J., Toom, M., Raidvee, A., Averin, K., \& Kreegipuu, K. (2013). An almost general theory of mean size perception. Vision Research, 83, 25-39. doi:10.1016/j.visres.2013.02.018

Alvarez, G. A. (2011). Representing multiple objects as an ensemble enhances visual cognition. Trends in Cognitive Sciences, 15(3), 122-131. doi:10.1016/j.tics.2011.01.003

Alvarez, G. A., \& Cavanagh, P. (2004). The capacity of visual short-term memory is set both by visual information load and by number of 
objects. Psychological Science: A Journal of the American Psychological Society, 15(2), 106-111. doi:10.1167/2.7.273

Alvarez, G. A., \& Franconeri, S. L. (2007). How many objects can you track? Evidence for a resource-limited attentive tracking mechanism. Journal of Vision, 7(13), 14.1-10. doi:10.1167/7.13.14

Alvarez, G. A., \& Oliva, A. (2008). the representation of simple ensemble visual features outside the focus of attention. Psychological Science, 19(4), 392-398. doi:10.1111/j.1467-9280.2008.02098.x

Ariely, D. (2001). Seeing sets: Representation by statistical properties. Psychological Science: A Journal of the American Psychological Society, 12(2), 157-162. doi:10.1111/1467-9280.00327

Ariely, D. (2008). Better than average? When can we say that subsampling of items is better than statistical summary representations? Perception \& Psychophysics, 70(7), 1325-1326. doi:10.3758/PP.70.7.1325

Attarha, M., \& Moore, C. M. (2015a). The capacity limitations of orientation summary statistics. Attention, Perception \& Psychophysics, 77(4), 1116-1131. doi:10.3758/s13414-015-0870-0

Attarha, M., \& Moore, C. M. (2015b). The perceptual processing capacity of summary statistics between and within feature dimensions. Journal of Vision, 15(4), 9. doi:10.1167/15.4.9

Attarha, M., Moore, C. M., \& Vecera, S. P. (2014). Summary statistics of size: Fixed processing capacity for multiple ensembles but unlimited processing capacity for single ensembles. Journal of Experimental Psychology: Human Perception and Performance, 40(4), 1440 1449. doi: $10.1037 / \mathrm{a} 0036206$

Awh, E., \& Jonides, J. (2001). Overlapping mechanisms of attention and spatial working memory. Trends in Cognitive Sciences, 5(3), 119 126. doi:10.1016/S1364-6613(00)01593-X

Awh, E., Jonides, J., \& Reuter-Lorenz, P. A. (1998). Rehearsal in spatial working memory. Journal of Experimental Psychology: Human Perception and Performance, 24(3), 780-790. doi:10.1037/00961523.24.3.780

Besner, D., Davies, J., \& Daniels, S. (1981). Reading for meaning: The effects of concurrent articulation. The Quarterly Journal of Experimental Psychology Section A, 33(4), 415-437. doi:10.1080/ 14640748108400801

Brady, T. F., \& Alvarez, G. A. (2011). Hierarchical encoding in visual working memory: Ensemble statistics bias memory for individual items. Psychological Science: A Journal of the American Psychological Society, 22(3), 384-392. doi:10.1177/ 0956797610397956

Bronfman, Z. Z., Brezis, N., Jacobson, H., \& Usher, M. (2014). We see more than we can report: "Cost free" color phenomenality outside focal attention. Psychological Science, 25(7), 1394-1403. doi:10. 1177/0956797614532656

Chong, S. C., Joo, S. J., Emmanouil, T.-A., \& Treisman, A. (2008). Statistical processing: Not so implausible after all. Perception \& Psychophysics, 70(7), 1327-1334. doi:10.3758/PP.70.7.1327

Chong, S. C., \& Treisman, A. (2003). Representation of statistical properties. Vision Research, 43, 393-404. doi:10.1016/S0042-6989(02)00596-5

Chong, S. C., \& Treisman, A. (2005a). Attentional spread in the statistical processing of visual displays. Perception \& Psychophysics, 67(1), 1-13. doi:10.3758/BF03195009

Chong, S. C., \& Treisman, A. (2005b). Statistical processing: Computing the average size in perceptual groups. Vision Research, 45, 891-900. doi: 10.1016/j.visres.2004.10.004

Cohen, J. (1994). The earth is round ( $\mathrm{p}<.05)$. American Psychologist, 49(12), 997-1003. doi:10.1037/0003-066X.49.12.997

Cohen, M. A., Dennett, D. C., \& Kanwisher, N. (2016). What is the bandwidth of perceptual experience? Trends in Cognitive Sciences, 20(5), 324-335. doi:10.1016/j.tics.2016.03.006

Cowan, N. (2001). The magical number 4 in short-term memory: A reconsideration of mental storage capacity. The Behavioral and Brain Sciences, 24(1), 87-114. Retrieved from http://www.ncbi. nlm.nih.gov/pubmed/11515286
De Fockert, J. W., \& Marchant, A. P. (2008). Attention modulates set representation by statistical properties. Perception \& Psychophysics, 70(5), 789-794. doi:10.3758/PP.70.5.789

Emmanouil, T.-A., \& Treisman, A. (2008). Dividing attention across feature dimensions in statistical processing of perceptual groups. Perception \& Psychophysics, 70(6), 946-954. doi:10.3758/PP.70.6.946

Haberman, J., Harp, T., \& Whitney, D. (2009). Averaging facial expression over time. Journal of Vision, 9(11). doi:10.1167/9.11.1

Haberman, J., \& Whitney, D. (2007). Rapid extraction of mean emotion and gender from sets of faces. Current Biology, 17(17). doi:10.1016/ j.cub.2007.06.039

Haberman, J., \& Whitney, D. (2009). Seeing the mean: Ensemble coding for sets of faces. Journal of Experimental Psychology: Human Perception and Performance, 35(3), 718-734. doi:10.1037/a0013899

Hochstein, S., \& Ahissar, M. (2002). View from the top: Hierarchies and reverse hierarchies in the visual system. Neuron, 36(5), 791-804. doi:10.1016/S0896-6273(02)01091-7

Huang L. (2015). Statistical properties demand as much attention as object features. PLoS ONE, 10(8), e0131191. doi: 10.1371/journal. pone. 0131191

Hubert-Wallander, B., \& Boynton, G. M. (2015). Not all summary statistics are made equal: Evidence from extracting summaries across time. Journal of Vision, 15(4), 1-12. doi:10.1167/15.4.5.doi

Im, H. Y., \& Chong, S. C. (2014). Mean size as a unit of visual working memory. Perception, 43, 663-676. doi:10.1068/p7719

Jackson-Nielsen, M., Cohen, M. A., \& Pitts, M. A. (2017). Perception of ensemble statistics requires attention. Consciousness and Cognition, 48, 149-160. doi:10.1016/j.concog.2016.11.007

Jeffreys, H. (1961). Theory of probability. Oxford: Oxford University Press.

Joo, S. J., Shin, K., Chong, S. C., \& Blake, R. (2009). On the nature of the stimulus information necessary for estimating mean size of visual arrays. Journal of Vision, 9(9. doi:10.1167/9.9.7

Kass, R. E., \& Raftery, A. E. (1995). Bayes factors. Journal of the American Statistical Association, 90(430), 773. doi:10.2307/2291091

Lamme, V. A., \& Roelfsema, P. R. (2000). The distinct modes of vision offered by feedforward and recurrent processing. Trends in Neurosciences, 23(11), 571-579. Retrieved from http://www.ncbi. nlm.nih.gov/pubmed/11074267

Luck, S. J., \& Vogel, E. K. (1997). The capacity of visual working memory for features and conjunctions. Nature, 390(6657), 279-281. doi: $10.1038 / 36846$

Marchant, A. P., Simons, D. J., \& De Fockert, J. W. (2013). Ensemble representations: Effects of set size and item heterogeneity on average size perception. Acta Psychologica, 142(2), 245-250. doi:10.1016/j. actpsy.2012.11.002

Masson, M. E. J. (2011). A tutorial on a practical Bayesian alternative to null-hypothesis significance testing. Behavior Research Methods, 679-690. doi:10.3758/s13428-010-0049-5

Maule, J., \& Franklin, A. (2016). Accurate rapid averaging of multihue ensembles is due to a limited capacity subsampling mechanism. Journal of the Optical Society of America A, 33(3), A22-29. doi: 10.1364/JOSAA.33.000A22

Maule, J., Witzel, C., \& Franklin, A. (2014). Getting the gist of multiple hues: Metric and categorical effects on ensemble perception of hue. Journal of the Optical Society of America A: Optics, Image Science, and Vision, 31(4), A93-A102. doi:10.1364/JOSAA.31.000A93

McNair, N. A., Goodbourn, P. T., Shone, L. T., \& Harris, I. M. (2016). Summary statistics in the attentional blink. Attention, Perception \& Psychophysics, 79(1), 100-116. doi:10.3758/s13414-016-1216-2

Myczek, K., \& Simons, D. J. (2008). Better than average: Alternatives to statistical summary representations for rapid judgments of average size. Perception \& Psychophysics, 70(5), 772-788. doi:10.3758/PP.70.5.772

Parkes, L., Lund, J., Angelucci, A., Solomon, J. A., \& Morgan, M. (2001). Compulsory averaging of crowded orientation signals in human vision. Nature Neuroscience, 4(7), 739-744. doi:10.1038/89532 
Pavlovskaya, M., Soroker, N., Bonneh, Y. S., \& Hochstein, S. (2015). Computing an average when part of the population is not perceived. Journal of Cognitive Neuroscience, 27(7), 1397-1411. doi:10.1162/ jocn_a_00791

Peirce, J. W. (2007). PsychoPy-Psychophysics software in Python. Journal of Neuroscience Methods, 162(1/2), 8-13. doi:10.1016/j. jneumeth.2006.11.017

Posner, M. I. (1980). Orienting of attention. Quarterly Journal of Experimental Psychology, 32(1), 3-25. doi:10.1080/ 00335558008248231

Pylyshyn, Z. W., \& Storm, R. W. (1988). Tracking multiple independent targets: Evidence for a parallel tracking mechanism. Spatial Vision, 3(3), 179-197. doi:10.1163/156856888X00122

Rensink, R. A., O'Regan, J. K., \& Clark, J. J. (1996). To see or not to see: The need for attention to perceive changes in scenes. Investigative Ophthalmology and Visual Science, 37(5), 322-326. doi:10.1111/j. 1467-9280.1997.tb00427.x

Rouder, J. N., Morey, R. D., Speckman, P. L., \& Province, J. M. (2012). Default Bayes factors for ANOVA designs. Journal of Mathematical Psychology, 56(5), 356-374. doi:10.1016/j. jmp.2012.08.001

Soto, D., Hodsoll, J., Rotshtein, P., \& Humphreys, G. W. (2008). Automatic guidance of attention from working memory. Trends in Cognitive Sciences, 12(9), 342-348. doi:10.1016/j.tics.2008.05.007

Treisman, A. (2006). How the deployment of attention determines what we see. Visual Cognition, 14(4/8), 411-443. doi:10.1080/ 13506280500195250
Utochkin, I. S., \& Tiurina, N. A. (2014). Parallel averaging of size is possible but range-limited: A reply to Marchant, Simons, and De Fockert. Acta Psychologica, 146, 7-18. doi:10.1016/j.actpsy.2013.11.012

Ward, E. J., Bear, A., \& Scholl, B. J. (2016). Can you perceive ensembles without perceiving individuals?: The role of statistical perception in determining whether awareness overflows access. Cognition, 152. doi:10.1016/j.cognition.2016.01.010

Watamaniuk, S. N. J., \& Duchon, A. (1992). The human visual system averages speed information. Vision Research, 32(5), 931-941. doi: 10.1016/0042-6989(92)90036-I

Whiting, B. F., \& Oriet, C. (2011). Rapid averaging? Not so fast! Psychonomic Bulletin \& Review, 18(3), 484-489. doi:10.3758/ s13423-011-0071-3

Wilkinson, L. (1999). Statistical methods in psychology journals: Guidelines and explanations. American Psychologist, 54(8), 594 604. doi:10.1037/0003-066X.54.8.594

Williams, D. W., \& Sekuler, R. (1984). Coherent global motion percepts from stochastic local motions. Vision Research, 24(1), 55-62. doi: 10.1016/0042-6989(84)90144-5

Woodman, G. F., \& Luck, S. J. (2004). Visual search is slowed when visuospatial working memory is occupied. Psychonomic Bulletin \& Review, 11(2), 269-274. doi:10.3758/BF03196569

Woodman, G. F., Vogel, E. K., \& Luck, S. J. (2001). Visual search remains efficient when visual working memory is full. Psychological Science, 12(3), 219-224. doi:10.1111/1467-9280.00339

Yamanashi Leib, A., Landau, A. N., Baek, Y., Chong, S. C., \& Robertson, L. (2012). Extracting the mean size across the visual field in patients with mild, chronic unilateral neglect. Frontiers in Human Neuroscience, 6, 1-11. doi:10.3389/fnhum.2012.00267 